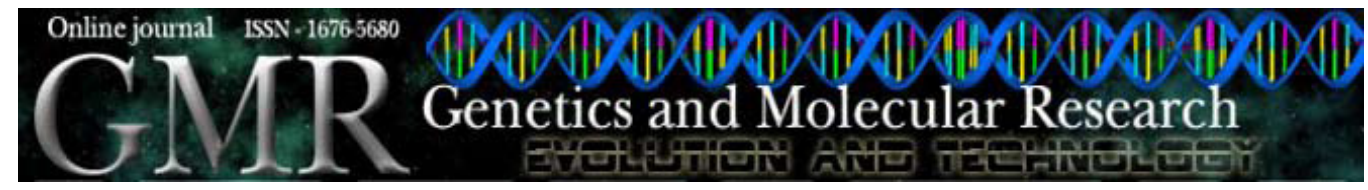

\title{
Molecular characterization of wheat germplasm using microsatellite markers
}

\author{
S. Ijaz and I.A. Khan \\ Centre of Agricultural Biochemistry and Biotechnology, \\ University of Agriculture, Faisalabad, Pakistan \\ Corresponding author: S. Ijaz \\ E-mail: siddraijaz_11@yahoo.com
}

Genet. Mol. Res. 8 (3): 809-815 (2009)

Received March 2, 2009

Accepted May 19, 2009

Published July 14, 2009

\begin{abstract}
We investigated the genetic diversity of 63 wheat genotypes, composed of 48 accessions and 15 varieties, using 56 polymorphic simple sequence repeat primers. One hundred and eighty-six loci were found, with a mean of 131.26 alleles per locus. Cluster analysis based on microsatellite allelic diversity discriminated the accessions and varieties into different clusters; genetic diversity was the highest between variety Kohistan-97 and accession number 011512 , giving a genetic similarity value of 0.4198 . Accession numbers 011484 and 011356 gave a genetic similarity value of 0.9589 , indicating that these accessions were $95.89 \%$ similar. We found that microsatellite markers could characterize and discriminate all of the genotypes; more primers could be used for saturation of different regions in further studies.
\end{abstract}

Key words: Triticum aestivum; Microsatellite; Molecular markers; Genome; Genetic distance; Population genetics 


\section{INTRODUCTION}

Common wheat Triticum aestivum $(2 \mathrm{n}=6 \mathrm{x}=42)$, belonging to the family Poaceae, which is the most diverse and important family of the plant kingdom, produces large edible grains and provides about one-half of humans' food calories and a large part of their nutrient requirements. Wheat (Triticum spp) is a worldwide cultivated and domesticated grass. The gradual increase in population demands a substantial increase in its productivity. Wheat has always been subjected to extensive and ceaseless research so as to maximize grain production but also to improve grain yield per unit area. However, there is still considerable room for improvement, especially to amplify efforts for continued genetic improvement of wheat to meet the growing requirements of an ever increasing population. Genetic manipulation is the best way to boost up wheat production. Therefore, it is necessary to estimate and study the genetic variation and mode of inheritance in different plant parameters to initiate productive wheat breeding programs. The stagnant yield of wheat in Pakistan is due to limited diversity in the germplasm used in breeding programs.

Through breeding and selection, great numbers of alleles have been lost, so that more difficulties have emerged for wheat improvement in modern agriculture systems (Allard, 1996; Hoisington et al., 1999). Molecular markers can provide detailed characterization of genetic resources. Molecular markers provide a direct measure of genetic diversity and go beyond the indirect diversity measures based on agronomic traits or geographic origin. Microsatellites are simple sequence repeats (SSR) of 1-6 nucleotides. They are abundant, dispersed throughout the genome and show higher levels of polymorphism than other genetic markers. These feature, coupled with their ease of detection, have made them useful markers. Their potential for automation and their inheritance in a co-dominant manner are additional advantages when compared with other types of molecular markers, and they cover all 21 wheat chromosomes. SSR markers have been used to characterize genetic diversity in wild relatives (Hammer et al., 2000) and in a seed bank collection of improved wheat germplasm (Börner et al., 2000; Huang et al., 2002). This study was conducted to estimate the genetic divergence among wheat accessions as well as cultivated varieties of Pakistan with the help of SSR markers. The present study addressed the utilization of microsatellite markers, to determine genetic diversity and relationships at the molecular level, among 63 genotypes of wheat. The genetic diversity and phylogenetic relationships determined in this study will help in the selection of parents to develop high-yielding varieties in breeding programs.

\section{MATERIAL AND METHODS}

\section{Plant materials/wheat germplasm}

Seeds of 63 genotypes (48 accessions and 15 varieties) were obtained: forty-eight wheat accessions collected from the Plant Genetic Resources Institute (PGRI), Islamabad, and 15 varieties of wheat, collected from Ayub Agricultural Research Institute (AARI), Faisalabad. All wheat accessions/cultivars were sown in small plastic pots in a growth chamber providing normal growing conditions. After two weeks, seedlings had grown. A 
total of 4-5 seedlings were cut and packed in plastic bags and stored at $-70^{\circ} \mathrm{C}$ for DNA extraction.

\section{DNA extraction and SSR analysis}

Genomic DNA of 63 wheat genotypes was extracted from fresh leaves, according to the method described by Khan et al. (2004). The quality and concentration of extracted DNA were estimated with a spectrophotometer. For SSR analysis, a total of 56 screened primer pairs were used for polymerase chain reaction (PCR) amplification. PCR conditions were maintained as described by Roder et al. (1998).

Each PCR was carried out in a $25-\mu \mathrm{L}$ reaction volume, containing $11.3 \mu \mathrm{L}$ double-

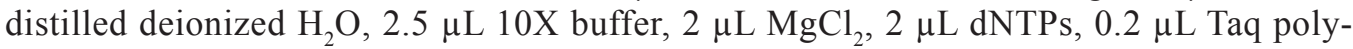
merase, $1 \mu \mathrm{L}$ of each primer of a primer pair, and $5 \mu \mathrm{L}$ DNA.

The SSR (PCR) amplification of genomic DNA was done by incubating the DNA samples at $94^{\circ} \mathrm{C}$ for $4 \mathrm{~min}$, then 45 cycles comprising $94^{\circ} \mathrm{C}$ for $1 \mathrm{~min}$, annealing of primer at $58-60^{\circ} \mathrm{C}$ for $1 \mathrm{~min}$ and then extension at $72^{\circ} \mathrm{C}$ for $1 \mathrm{~min}$. The final extension was carried out at $72^{\circ} \mathrm{C}$ for $10 \mathrm{~min}$.

The PCR products were electrophoresed on $3 \%$ agarose gels containing $7 \mu \mathrm{L}$ ethidium bromide, at $80 \mathrm{~V}$ for $1 \mathrm{~h}$, and observed under a UV transilluminator.

Bands were counted and the presence and absence of bands were scored as 1 and 0 , respectively. The data were collected and aligned for the construction of cluster analysis. The cluster analysis of 63 genotypes was performed using the POP Gen software version 1.32 (Yeh et al., 2000) to determine genetic diversity and similarity among accessions.

\section{RESULTS}

Fifty-six screened polymorphic SSR primer pairs were used to assess the extent of genetic diversity among 63 wheat genotypes. These 56 SSR primer pairs indicated 186 loci and all were shown to be polymorphic. A total of 7351 alleles were identified and the number of alleles detected at a single locus ranged from 0 (Xgwm 112-3B) to 368 (Xgwm 63-7A) with a mean of 131.26 alleles per locus. More alleles were observed in B genome as compared to A and D genomes. The SSR markers showed a high level of polymorphism ranging from $10.52 \%$ (Xgwm 162-3A) to $98.42 \%$ (Xgwm 111-7D). To examine the genetic relationship between 63 wheat genotypes, a dendogram was constructed (Figure 1). Cluster analysis indicated that 63 genotypes could be divided into three major clusters. Cluster I included all 15 varieties, cluster II was the largest and included 25 accessions of 48 and cluster III comprised the remaining 20 accessions. Three accessions were distinct, namely 011350, 011351, and 011352, and of these three, accessions 011351 and 011352 are closely related, 011350 is separate, and these three accessions may be used in a breeding program as distinct parents. A maximum genetic similarity value of 0.9589 was observed between accession numbers 011484 and 011356 , which revealed a high degree of similarity to the extent of $95.89 \%$ existing between them. Whereas, a minimum genetic similarity value of 0.4198 was observed between the variety Kohistan-97 and accession number 011512 , which showed that they are $41.98 \%$ dissimilar. 


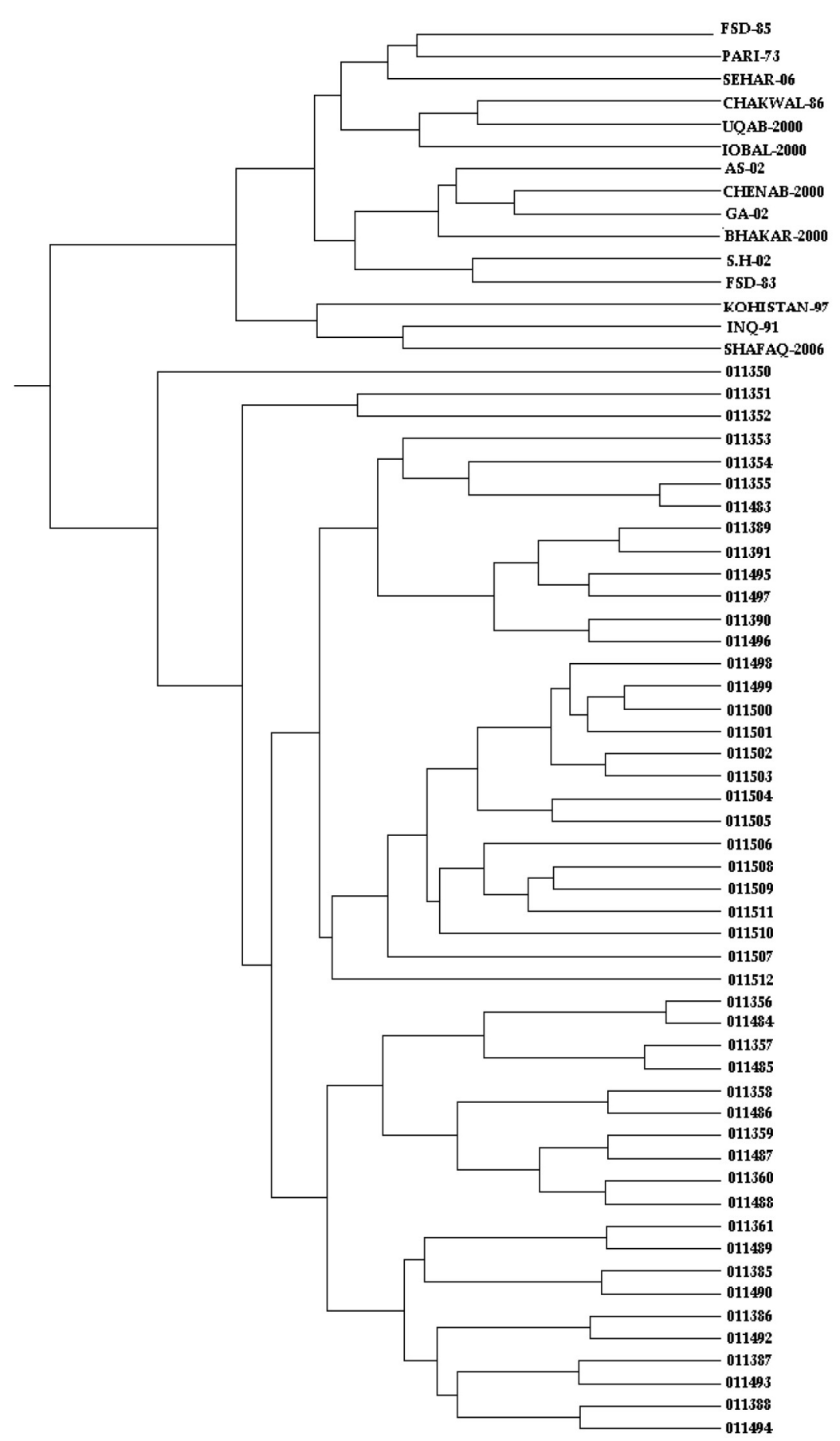

Figure 1. Overall dendogram showing 63 genotypes, based on Nei’s original measures (1972). 


\section{DISCUSSION}

The characterization of genetic diversity within a closely related crop germplasm is an essential tool for rational use of genetic resources. The analysis of genetic variation in breeding materials is of fundamental interest to plant breeders, as it contributes to selection, monitoring of germplasm and prediction of potential genetic gain (Chakravarthy and Naravaneni, 2006). Molecular markers have the potential to detect genetic diversity and to aid in the management of plant genetic resources (Ford-Lloyd et al., 1997; Virk et al., 1995; Song et al., 2003). Among various molecular markers currently available, SSR or microsatellite markers are often chosen as the preferred markers for a variety of applications in breeding because of their multi-allelic nature, co-dominant inheritance, relative abundance, and extensive genome coverage (Gupta and Varshney, 2000). Microsatellite markers are becoming the markers of choice due to the level of polymorphism, as well as higher reliability (Plaschke et al., 1995; Fu et al., 2005). In wheat, abundant wheat genomic SSR markers are now available and have been mapped (Roder et al., 1998), making them a useful resource for further studies. Microsatellite markers are useful and becoming popular for different applications in wheat breeding due to their high level of polymorphism and easy handling (Devos et al., 1995; Roder et al., 1995; Bryan et al., 1997; Roy et al., 1999; Lelley et al., 2000) and are used to evaluate genetic diversity of hexaploid wheat (Triticum aestivum L.) landraces in relation to their geographic origin (Al Khanjari et al., 2007). The present study addressed the utility of SSR markers in revealing assessment of genetic variability and diversity at the molecular level among 63 wheat genotypes wherein 56 screened polymorphic SSR primers were used, which were earlier identified in the genomic regions of A, B, and D genomes of wheat. The SSR marker loci generated by the 56 primer pairs were used to assess the genetic diversity among 63 wheat genotypes. The microsatellite or SSR primers generated 7351 alleles with the number of alleles per locus varying from 0 to 368 . This study also showed that primer pair Xgwm 63-7A generated a maximum number of 368 bands while primer pair Xgwm $112-3 \mathrm{~B}$ produced a minimum number of bands $(0)$. This revealed significant differences in allelic diversity among various microsatellite loci. Many studies have also reported remarkable differences in allelic diversity among various microsatellite loci (Akagi et al., 1997; McCouch et al., 2001; Ravi et al., 2003; Ram et al., 2007). The alleles revealed by markers showed a higher degree of polymorphism. More alleles were identified in genome B (a total of 3069 alleles) as compared to A and D genomes (genome A contained a total of 2457 alleles and genome D contained a total of 1825 alleles). This confirmed that there was a higher polymorphism level in the B genome. Similar observation for higher polymorphism level of genome B was also reported by Wang et al. (2007). The present observations also agree with the results of studies by Cho et al. (2000) in rice, Scott et al. (2000) in grapes and Eujayl et al. (2002) in wheat.

The 63 genotypes, which consisted of 48 accessions collected from PGRI, Islamabad, and 15 varieties of wheat from AARI, Faisalabad, were further subjected to cluster analysis. Cluster analysis of the SSR-based genetic similarity matrix resulted in the classification of accessions and varieties into separate clusters. The dendogram resulting from cluster analysis revealed 3 clusters. Cluster I included all 15 varieties, cluster II was the largest and included 25 accessions of 48, and cluster III comprised the remaining 20 accessions. Three accessions were distinct, namely 011350,011351 , and 011352 , and of these three, two accessions, 011351 and 011352 , are closely related, 011350 is separate, and these three accessions may be used in breeding programs as distinct parents. 
A maximum genetic similarity value of 0.9589 was observed between the accession numbers 011484 and 011356 , which revealed a high degree of similarity to the extent of $95.89 \%$ existing between them. A minimum genetic similarity value of 0.4198 was observed between the variety Kohistan-97 and accession number 011512 , which showed that they are $41.98 \%$ dissimilar. Similar studies were conducted by different investigators using SSR markers (Panaud et al., 1996; Chakravarthy and Naravaneni, 2006; Ram et al., 2007). It was assumed that such a high level of genetic similarity may be the result of biased selection of the material in the previous breeding programs, which ultimately narrowed the genetic base of the wheat germplasm in the country. It is further suggested that more polymorphic wheat microsatellites could be used for efficient screening of the germplasm by saturating more regions of the wheat genome.

\section{REFERENCES}

Akagi H, Yokozeki Y, Inagaki A and Fujimurati T (1997). Highly polymorphic microsatellites of rice consist of AT repeats, and a classification of closely related cultivars with these microsatellite loci. Theor. Appl. Genet. 94: 61-67.

Al Khanjari S, Hammer K, Buerkert A and Röder MS (2007). Molecular diversity of Omani wheat revealed by microsatellites: II. Hexaploid landraces. Genet. Resour. Crop Evol. 54: 1407-1417.

Allard RW (1996). Genetic basis of the evolution of adaptedness in plants. Euphytica 92: 1-11.

Börner A, Chebotar S and Korzun V (2000). Molecular characterization of the genetic integrity of wheat (Triticum aestivum L.) germplasm after long-term maintenance. Theor. Appl. Genet. 100: 494-497.

Bryan GJ, Collins AJ, Stephenson P, Orry A, et al. (1997). Isolation and characterisation of microsatellites from hexaploid bread wheat. Theor. Appl. Genet. 94: 557-563.

Chakravarthi BK and Naravaneni R (2006). SSR marker based DNA fingerprinting and diversity study in rice (Oryza sativa. L). Afr. J. Biotechnol. 8: 684-688.

Cho YG, Ishii T, Temnykh S, Chen X, et al. (2000). Diversity of microsatellites derived from genomic libraries and GenBank sequences in rice (Oryza sativa L.). Theor. Appl. Genet. 100: 713-722.

Devos KM, Bryan GJ, Collins AJ, Stephenson P, et al. (1995). Application of two microsatellite sequences in wheat storage proteins as molecular markers. Theor. Appl. Genet. 90: 247-252.

Eujayl I, Sorrells ME, Baum M, Wolters P, et al. (2002). Isolation of EST-derived microsatellite markers for genotyping the A and B genomes of wheat. Theor. Appl. Genet. 104: 399-407.

Ford-Lloyd BV, Jackson MT and Newbury HJ (1997). Molecular Markers and the Management of Genetic Resources in Seed Genebanks: A Case Study of Rice. In: Biotechnology and Plant Genetic Resources - Conservation and Use (Callow JA, Ford-Lloyd BV and Newbury HJ, eds.). CAB International (Centro de Biociência Agrícola Internacional), Wallingford, 103-118.

Fu YB, Peterson GW, Richards KW, Somers D, et al. (2005). Allelic reduction and genetic shift in the Canadian hard red spring wheat germplasm released from 1845 to 2004. Theor. Appl. Genet. 110: 1505-1516.

Gupta PK and Varshnei RK (2000). The development and use of microsatellite markers for genetic analysis and plant breeding with emphasis on bread wheat. Euphytica 113: 163-185.

Hammer K, Filatenko AA and Korzun V (2000). Microsatellite markers - a new tool for distinguishing diploid wheat species. Genet. Resour. Crop Evol. 47: 497-505.

Hoisington D, Khairallah M, Reeves T, Ribaut JM, et al. (1999). Plant genetic resources: what can they contribute toward increased crop productivity? Proc. Natl. Acad. Sci. U. S. A. 96: 5937-5943.

Huang Q, Borner A, Roder S and Ganal W (2002). Assessing genetic diversity of wheat (Triticum aestivum L.) germplasm using microsatellite markers. Theor. Appl. Genet. 105: 699-707.

Khan IA, Awan FS, Ahmad A and Khan AA (2004). A modified mini-prep method for economical and rapid extraction of genomic DNA in plants. Plant Mol. Biol. Rep. 22: 89a-89e.

Lelley T, Stachel M, Grausgruber H and Vollmann J (2000). Analysis of relationships between Aegilops tauschii and the D genome of wheat utilizing microsatellites. Genome 43: 661-668.

McCouch SR, Temnykh S, Lukashova A, Coburn J, et al. (2001). Microsatellite Markers in Rice: Abundance, Diversity and Applications. In: Rice Genetics IV. International Rice Research Institute (IRRI), Manila, 117-135.

Nei M (1972). Genetic distances between populations. Am. Nat. 106: 283-292.

Panaud O, Chen X and McCouch SR (1996). Development of microsatellite markers and characterization of simple 
sequence length polymorphism (SSLP) in rice (Oryza sativa L.). Mol. Gen. Genet. 252: 597-607.

Plaschke J, Ganal MW and Röder MS (1995). Detection of genetic diversity in closely related bread wheat using microsatellite markers. Theor. Appl. Genet. 91: 1001-1007.

Ram SG, Thiruvengadam V and Vinod KK (2007). Genetic diversity among cultivars, landraces and wild relatives of rice as revealed by microsatellite markers. J. Appl. Genet. 48: 337-345.

Ravi M, Geethanjali S, Sameeyafarheen F and Maheswaran M (2003). Molecular marker based genetic diversity analysis in rice (Oryza sativa L.) using RAPD and SSR markers. Euphytica 133: 243-252.

Roder MS, Plaschke J, Konig SU, Borner A, et al. (1995). Abundance, variability and chromosomal location of microsatellites in wheat. Mol. Gen. Genet. 246: 327-333.

Roder MS, Korzun V, Wendehake K, Plaschke J, et al. (1998). A microsatellite map of wheat. Genetics 149: 2007-2023.

Roy JK, Prasad M, Varshney RK, Balyan HS, et al. (1999). Identification of a microsatellite on chromosomes 6B and a STS on 7D of bread wheat showing an association with preharvest sprouting tolerance. Theor. Appl. Genet. 99: 336-340.

Scott KD, Eggler P, Seaton G, Rossetto M, et al. (2000). Analysis of SSRs derived from grape ESTs. Theor. Appl. Genet. 100: 723-726.

Song ZP, Xu X, Wang B, Chen JK, et al. (2003). Genetic diversity in the northernmost Oryza rufipogon populations estimated by SSR markers. Theor. Appl. Genet. 107: 1492-1499.

Virk PS, Ford-Lloyd BV, Jackson MT and Newbury HJ (1995). Use of RAPD for the study of diversity within plant germplasm collections. Heredity 74 (Pt 2): 170-179.

Wang HY, Wei YM, Yan ZH and Zheng YL (2007). EST-SSR DNA polymorphism in durum wheat (Triticum durum L.) collections. J. Appl. Genet. 48: 35-42.

Yeh FC, Yang RC, Boyle TBJ, Ye Z, et al. (2000). PopGene32, Microsoft Windows-Based Freeware for Population Genetic Analysis. Version 1.32. Molecular Biology and Biotechnology Centre, University of Alberta, Edmonton. 